\title{
The EUROSTROKE cohorts: a short description and data analytical approach
}

\section{L Bots, P C Elwood, Y Nikitin, J T Salonen, A Freire de Concalves, D Inzitari, J Sivenius, A Trichopoulou, J Tuomilehto, P J Koudstaal, D E Grobbee}

J Epidemiol Community Health 2002;56(Suppl I):i2-i7

This paper describes the design and methodology of the participating cohorts in the EUROSTROKE project.

Information is given about the cohort sampling, its size, the follow up procedures and event classification. Information is also given about the measurement of the cardiovascular and cerebrovascular risk factors in each of the cohorts separately. The cohorts described are the Caerphilly study in Cardiff, United Kingdom; the Kuopio Ischaemic Heart disease study in Kuopio, Finland; the Portugal study in Coimbra, Portugal; the EPIC cohort in Athens, Greece; the llsa study from Firenze, Italy; the Rotterdam Study in Rotterdam, the Netherlands, and the Novosibirsk cohort in Novosibirsk, Russia.

See end of article for authors' affiliations

Correspondence to: $\operatorname{Dr} M$ L Bots, Julius Centre for General Practice and Patient Oriented Research, University Medical Centre Utrecht, room D01.335, Heidelberglaan 100, 3584 CX Utrecht/3508 GA Utrecht, the Netherlands: M.L.Bots@jc.azu.nl

Accepted for publication 30 July 2001
E UROSTROKE is a collaborative study among European research centres to investigate the variation in incidence of fatal and non-fatal ischaemic and haemorrhagic stroke among populations in different European countries and to evaluate whether the observed differences in stroke incidence across countries can be explained by differences in prevalence of established cardiovascular risk factors and to study the relative importance of smoking and some selected dietary factors (potassium intake, alcohol consumption), haemostatic disturbances (fibrinogen) and comorbidity (rheumatic heart disease, atrial fibrillation) compared with established risk factors as determinants of the occurrence of ischaemic and haemorrhagic stroke (fig 1). ${ }^{1}$ The EUROSTROKE database is drawn from ongoing European population-based prospective follow up studies (cohorts) and is designed as a casecontrol study nested within these ongoing studies. For each stroke case, two controls were sampled. Controls were matched on day of baseline examination only. Apart from its objectives, the EUROSTROKE database allows for aetiological analyses looking into various risk factors for stroke. EUROSTROKE formally started on 1 January 1994. At present, data on stroke and risk factors of four cohorts were available for analyses.

As EUROSTROKE is based on ongoing cohort studies, information on cardiovascular risk factors in each of the participating centres was already being collected before the EUROSTROKE project was established. Whenever possible, an exhaustive attempt was made to further harmonise the collected information to make comparison across studies possible. Nevertheless, baseline measure- ments could not be standardised beyond the attempts done in each individual study a priori.

This paper describes the individual cohorts in detail (table 1).

\section{DESCRIPTION OF THE COHORTS \\ Cardiff, United Kingdom}

The British contribution to EUROSTROKE comes from the Caerphilly Heart Disease study in Wales, United Kingdom, in which 2512 men, aged 45 to 59 years are participating. ${ }^{2}$ Baseline examinations took place from 1979 to 1983. Follow up examinations were performed from 1984 to 1988 (phase II) and from 1989 to 1993 (phase III). Stroke events were registered through national mortality statistics, hospital discharge records, self report and family report. Of the registered events, additional information on signs and symptoms, on neuroimaging, necropsy and a copy of the discharge records were collected. When complete, stroke cases were submitted for review to the EUROSTROKE case review board. Recently, 100 stroke events had been submitted for review and 200 controls subjects had been drawn from the remaining cohort. Eighty four stroke cases were classified as definite/probable stroke by the EUROSTROKE review board; those were contributed to the EUROSTROKE database.

Information on smoking, alcohol consumption, and medical history was obtained by questionnaire. The subject's smoking behaviour was categorised into current, former or never. Alcohol consumption was categorised into current drinkers and non-current drinkers (former and never). In addition, an estimate of grams of alcohol per day was obtained. Presence of diabetes mellitus was based on the question: "Do you suffer from diabetes mellitus?". Information on a history of stroke was obtained by direct questioning at baseline: "Did you ever suffer from a stroke?". A similar approach was taken for myocardial infarction. Presence of angina pectoris was based on either the cardiovascular Rose questionnaire or direct questioning. Systolic and diastolic blood pressure were measured once at one occasion in sitting position. Height and weight were measured and body mass index (kg/ $\mathrm{m}^{2}$ ) was calculated. An electrocardiogram was made and the presence of atrial fibrillation and of a Q-wave myocardial infarction and left ventricular hypertrophy were assessed according to the Minnesota classification system. Left ventricular hypertrophy was assessed according to the Minnesota classification system (codes 3.1, 3.3, 


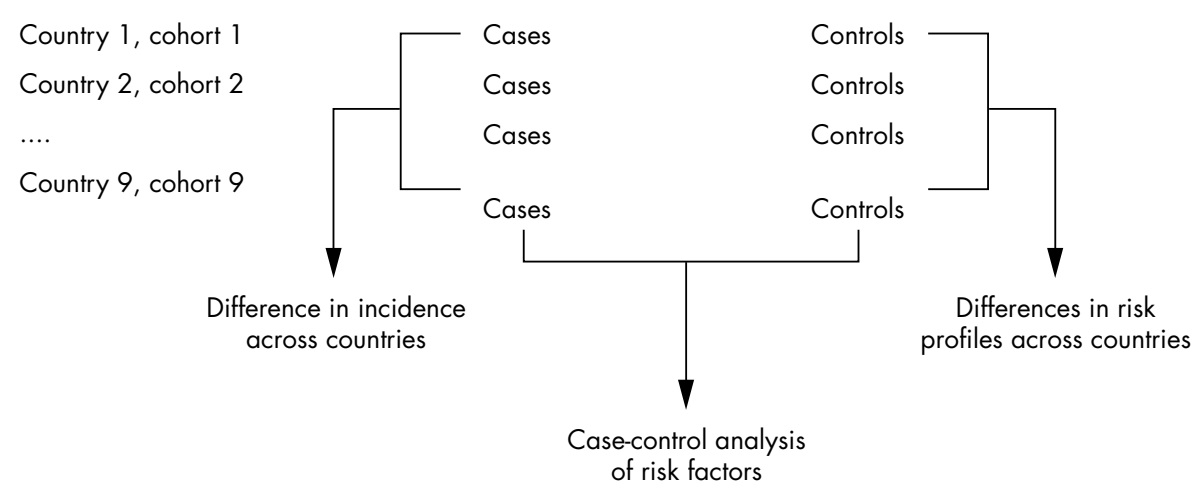

Figure 1 EUROSTROKE: Schematic presentation of the approach to answer the three study objectives.

of risk factors

Table 1 Information on sampling frame, sampling method, sample size and procedures for follow up for each of the participating centres

\begin{tabular}{|c|c|c|c|c|c|c|}
\hline Country & Site & $\begin{array}{l}\text { Principal } \\
\text { investigator }\end{array}$ & $\begin{array}{l}\text { Sample } \\
\text { frame }\end{array}$ & Sample method & $\begin{array}{l}\text { Sample } \\
\text { size }\end{array}$ & Follow up design \\
\hline \multirow[t]{2}{*}{ Finland } & Kuopio & J T Salonen & $\begin{array}{l}\text { General } \\
\text { population }\end{array}$ & $\begin{array}{l}\text { Age stratified random sample of } \\
\text { men, aged } 42,48,54 \text { and } 60 \text { years }\end{array}$ & 2682 & $\begin{array}{l}\text { National mortality registry } \\
\text { Stroke registry }\end{array}$ \\
\hline & Helsinki & J Tuomilehto & $\begin{array}{l}\text { General } \\
\text { population }\end{array}$ & Random sample & around 15000 & $\begin{array}{l}\text { National mortality registry } \\
\text { Stroke registry }\end{array}$ \\
\hline Greece & Athens & A Trichopoulou & $\begin{array}{l}\text { General } \\
\text { population }\end{array}$ & $\begin{array}{l}\text { Men and women volunteers, 30-75 } \\
\text { years }\end{array}$ & 28577 & $\begin{array}{l}\text { National mortality registry } \\
\text { Active follow up }\end{array}$ \\
\hline Italy & Firenze & D Inzitari & $\begin{array}{l}\text { General } \\
\text { population }\end{array}$ & $\begin{array}{l}\text { Age stratified random sample men } \\
\text { and women, aged } 65-84 \text { years }\end{array}$ & 4373 & $\begin{array}{l}\text { Hospital-based stroke registry } \\
\text { Follow up by questionnaire, } \\
\text { telephone and re-examination }\end{array}$ \\
\hline Netherlands & Rotterdam & $\begin{array}{l}\text { D E Grobbee } \\
\text { P J Koudstaal }\end{array}$ & $\begin{array}{l}\text { General } \\
\text { population }\end{array}$ & $\begin{array}{l}\text { Random sample of men and women, } \\
\text { aged } 55 \text { years or over }\end{array}$ & 7983 & $\begin{array}{l}\text { National mortality registry } \\
\text { General Practitioner Stroke } \\
\text { Registry }\end{array}$ \\
\hline Portugal & Coimbra & A Freire Concalves & $\begin{array}{l}\text { General } \\
\text { population }\end{array}$ & $\begin{array}{l}\text { Random sample of men and women, } \\
\text { aged } 55 \text { years or over }\end{array}$ & 3020 & $\begin{array}{l}\text { General Practitioner based } \\
\text { stroke registry }\end{array}$ \\
\hline Russia & Novosibirsk & Y Nikitin & $\begin{array}{l}\text { General } \\
\text { population }\end{array}$ & $\begin{array}{l}\text { Random sample of men and women, } \\
\text { aged } 25 \text { to } 64 \text { years }\end{array}$ & 9006 & $\begin{array}{l}\text { Stroke Registry (MONICA } \\
\text { procedures) }\end{array}$ \\
\hline United Kingdom & Cardiff & P C Elwood & $\begin{array}{l}\text { General } \\
\text { population }\end{array}$ & $\begin{array}{l}\text { Random sample of men, aged } \\
45-59 \text { years }\end{array}$ & 2512 & $\begin{array}{l}\text { National mortality registry } \\
\text { Hospital records } \\
\text { Follow up by questionnaire }\end{array}$ \\
\hline
\end{tabular}

3.4). ${ }^{3}$ A fasting blood sample was taken for determination of serum lipids (total cholesterol, HDL cholesterol). Serum total cholesterol level was determined with an automatic analyser using enzymatic procedures. ${ }^{4}$ Fibrinogen was measured according to the nephelometrical method.

In the Caerphilly Study a self administered, semiquantitative 56 item food frequency questionnaire was given to each participant for completion, with help from his partner, at home. In addition, after receiving instructions by nutrionists, a representative subsample (3 in 10) was asked to complete a weighed seven day record. Food intake was estimated from the food frequency questionnaire by multiplying the frequency of consumption by average portion size and nutrient intake was calculated. ${ }^{5}$

\section{Kuopio, Finland}

The Finnish contribution to EUROSTROKE comes from the Kuopio Ischaemic Heart Disease Risk Factor study, which is a population-based prospective cohort study comprised of an age stratified random sample of 2682 men aged 42, 48, 54 and 60 years. The baseline examination was performed between 1984 and 1989. ${ }^{6}$ Fatal and non-fatal stroke cases were collected through the national mortality statistics and the FINMONICA stroke registries. Stroke was defined according to FINMONICA criteria and definitions. ${ }^{7}$ Case ascertainment from the baseline examination to 1 January 1993 revealed 74 stroke cases. Control subjects $(n=148)$ were randomly drawn from the cohort that remained free from stroke during follow up.

Information on smoking, alcohol consumption, and medial history was obtained by questionnaire. The subject's smoking behaviour was categorised into current, former or never. Alcohol consumption was categorised into current drinkers and non-current drinkers (former and never). In addition, an estimate of grams of alcohol per day was obtained. Presence of diabetes mellitus was based on the question: "Do you suffer from diabetes mellitus?". Information on a history of stroke was obtained by direct questioning at baseline: "Did you ever suffer from a stroke?" A similar approach was taken for myocardial infarction. Presence of angina pectoris was based on either the cardiovascular Rose questionnaire or direct questioning.

Systolic and diastolic blood pressure were measured twice at one occasion in sitting position. Height and weight were measured and body mass index $\left(\mathrm{kg} / \mathrm{m}^{2}\right)$ was calculated. An electrocardiogram was made and the presence of atrial fibrillation and of a Q-wave myocardial infarction and left ventricular hypertrophy were assessed according to the Minnesota classification system. Left ventricular hypertrophy was assessed according to the Minnesota classification system (codes 3.1, 3.3, 3.4). A fasting blood sample was taken for determination of serum lipids (total cholesterol, HDL cholesterol). Serum total cholesterol level was determined with an automatic analyser using enzymatic procedures. Fibrinogen was measured according to the Von Clauss method. ${ }^{8}$

In the Kuopio Ischaemic Heart Disease Risk factor study the consumption of food was assessed at time of blood sampling with an instructed and interview checked four day food recording by household measures, as described elsewhere. ${ }^{9}$ The nutrient intake was estimated using the Nutrica computer program for calculation of nutrient intake. 


\section{Athens, Greece}

The Greek contribution to EUROSTROKE comprises data from the Greek component of the European Prospective Investigation into Cancer and nutrition (EPIC). ${ }^{10}$ The EPIC study is a large prospective study, aiming at the investigation of the role of nutrition and related factors on the aetiology of cancer and other chronic diseases. The study is being carried out in nine European countries, under the coordination of the International Agency for Cancer Research (IARC) with the support of the "Europe Against Cancer Program" of the European Union combined with national and IARC resources.

In Greece, EPIC is being carried out by the Department of Hygiene and Epidemiology of the University of Athens Medical School, in collaboration with the Department of Nutrition and Biochemistry of the National School of Public Health and the Greek Society of Nutrition and Foods. The Greek cohort consists of 28577 volunteers, men and women aged 30-75 years, residing in urban and rural areas from all over Greece. Recruitment was completed in March 1999 and the follow up of study subjects started in January 1997. The follow up will last for approximately 10 years and it will be conducted at two year intervals from the date of recruitment.

Information on medical history, lifestyle and environmental factors is provided by the study participants, by means of an interview, conducted by trained personnel. All data are recorded in standardised questionnaires. History of stroke, rheumatic heart disease, myocardial infarction, angina pectoris, diabetes mellitus and hypertension is recorded. Family history of stroke and myocardial infarction, socioeconomic indices, such as educational level and occupation, as well as history of tobacco smoking and physical activity, are also recorded. Dietary intake, supplementation and alcohol consumption are assessed via a validated semi-quantitative food frequency questionnaire administered by trained interviewers.

Anthropometric indices like height, weight, waist and hip circumferences are measured using standardised procedures. Arterial blood pressure is measured twice, with the use of a mercury sphygmomanometer. All drugs consumed the week before recruitment (especially type and quality of hypertension treatment), are recorded and coded according to the criteria of the Anatomical Therapeutic Chemical (ATC) classification index of medications. Finally, a non-fasting venipuncture is performed. Blood samples are stored in liquid nitrogen containers for future analyses.

Active follow up is implemented in the Greek centre. Record linkage with the National Death Registration System is not possible because of confidentiality reasons. Therefore, fatal stroke cases are being ascertained actively, by tracing the death certificates in regional mortality registries. In addition, fatal cases are verified through medical records and necropsy reports (whenever performed). Coding of fatal strokes is done according to the 10th revision of the International Classification of Diseases. With respect to non-fatal stroke cases, efforts are made to achieve the highest level of confirmation. A stroke case ascertainment questionnaire has been developed, collecting information on signs, symptoms (location, onset, duration), laboratory findings and treatment. Hospital discharge diagnoses are recorded and copies of reports of computed tomography, magnetic resonance imaging, angiograms, or lumbar punctures are being collected, whenever possible. In case of non-hospitalisation, information is obtained by contacting the treating physician. All stroke cases, especially the non-hospitalised ones, will be evaluated by a neurologist and subsequently submitted for review to the EUROSTROKE review board.

The Greek EPIC centre has provided data on cerebrovascular risk factor profiles (gender, age, systolic blood pressure, antihypertensive treatment, pulse rate, current smoking, history of stroke, myocardial infarction, angina pectoris, diabetes mellitus, height, weight, body mass index) for a sample of the study population. This sample consists of the 2659 participants recruited during 1994.

\section{Key points}

- Differences in stroke mortality across European countries are impressive.

- Explanation of these differences is important: incidence or case fatality.

- EUROSTROKE aims at explaining differences in stroke mortality, using data from existing cohorts in Europe.

\section{ILSA study, Italy}

The Italian contribution to EUROSTROKE arises from the Italian Longitudinal Study on Ageing (ILSA). ILSA is a population-based, multicentre prospective cohort study focusing at normal aging and aging disease and its determinants. The ILSA is described in detail elsewhere. ${ }^{11}$ In short the ILSA is aimed at the evaluation of age related functional changes of cardiovascular, endocrine and nervous systems, and at the estimation, in a cohort of older Italians, of prevalence and incidence rates of the following age associated diseases: angina pectoris, myocardial infarction, cardiac arrhythmia, congestive heart failure, hypertension, peripheral artery disease, diabetes, stroke, dementia, parkinsonism and distal symmetric neuropathies. A random sample of 5632 people aged 65-84 years, free dwelling or institutionalised, stratified by age and gender, was extracted from the demographic lists in eight Italian municipalities: Genoa, Segrate (Milan), Selvazzano-Rubano (Padua), Impruneta (Florence), Fermo (Ascoli Piceno), Naples, Casamassima (Bari), and Catania. Following the equal allocation strategy, 88 subjects of each gender were included in four age groups $(65-69,70-74,75-79$, $80-84$ years) in every participating centre, yielding the overall study sample.

The ILSA design includes both cross sectional and longitudinal components. At the baseline survey, cases were identified through a two phase design. In the screening phase (phase 1), involving all the participants in the sample, a personal interview was conducted to determine demographic characteristics and work category, and to collect information on functional conditions, risk factors and diseases symptoms; a physical examination, carried out by a general physician, and laboratory investigations including haematology tests, spirometry, electrocardiography and retinography were performed. Data on anthropometry and nutrition were also collected. In phase 2 a specialist (geriatrician or neurologist, according to the different conditions) confirmed, through a visit and review of medical records, disease diagnoses in suspected cases.

The reliability of the diagnostic process was assured by the following methods: (1) interviewers, general physicians, neurologists and geriatricians were centrally trained and certified in data collection and form completion procedures; (2) site visits to all participating centres were performed during the data collection phase by members of the Coordinating Unit located in Florence, in order to supervise the field work, step by step, and to discuss potential problems with the staff members of each centre; (3) the overall diagnostic process was centrally reviewed by a panel of experts for each disease. The cross sectional survey was carried out between March 1992 and June 1993 (prevalence day: I March 1992).

According to the ILSA methodology, the screening of suspected stroke cases was based on a comprehensive home interview, performed by trained lay personnel using a structured questionnaire, and on a simple neurological examination made by the general physician. The questionnaire contained: (1) a simple question: have you ever had a diagnosis of stroke or cerebral haemorrhage?, and, independently from this one, (2) questions about previous symptoms: have you ever had for more than 24 hours: (a) speech disturbances; (b) hemifacial palsy or twisted mouth; (c) limb weakness or 
paralysis? Subjects answering "yes" to any of the four questions were invited for phase 2 examination. If the subject was unable to cooperate in the interview, for example, because of cognitive impairment, information was gathered from a proxy responder. With a simple neurological examination gait, limbs tone and power, tendon reflexes, touch and pain sensation were evaluated. Subjects presenting with a deficit in limbs power were also invited for further examination.

Subjects screened as possible stroke cases were visited by a neurologist who, in a detailed and standardised fashion, inquired about symptoms, evaluated gait, speech and language, cranial nerves, limbs tone and power, tendon reflexes, cerebellar function and sensation, and reviewed medical records. According to the World Health Organisation, stroke was defined as "rapidly developing clinical signs of focal or global disturbance of cerebral function, with symptoms lasting for more than 24 hours or leading to death, with no apparent cause other than of vascular origin". Both first ever and recurrent strokes were considered, while transient ischaemic episodes were not taken into account. Date of stroke, initial symptoms and signs, vascular risk factors, hospital admission, laboratory investigations, treatment, and current level of disability were registered. Information on subjects refusing phase 2 examination as well as on subjects dead between phase 1 and phase 2 was gathered by interviewing general practitioners (GP), and reviewing medical records.

Follow up examination of the ILSA participants started on September 1995. Participants were re-examined following the same procedures used at the baseline examination. Briefly, the case identification strategy consisted of a screening phase, administered by lay interviewers to all participants, and a clinical assessment of those who screened positive, carried out by specialists (neurologists and geriatricians), to make the final diagnoses. During this second cross sectional survey, for every deceased individual, medical information was gathered from both the subject's GP and relatives, using ad hoc questionnaires.

Moreover, two years after the baseline survey the overall study population had been followed up by telephone interview to determine the vital status and hospitalisation. Information regarding people who either had died or were unable to answer the interview had been gathered from both GP and relatives. A clinical investigator in each study centre interviewed the GP of every deceased subject about the possible occurrence of a stroke before death. Information about drug prescriptions, other diseases, hospitalisation, and institutionalisation was also gathered from GP as well as from subject's relatives. Hospital discharge diagnoses and death certificate diagnoses were reviewed for each hospitalised or deceased subject.

\section{Coimbra, Portugal}

The Portugese contribution to EUROSTROKE is based on a single centre prospective follow up study in a population random sample of 3020 people of 50 years or over living in the district of Coimbra; Coimbra Incidence Stroke Study . Baseline data were collected from November to December 1996 by local general practitioners.

Medical history was taken according to a EUROSTROKE standard questionnaire and physical and laboratory measurements were made. Hypertension was diagnosed if present in medical records, if the patient was under specific antihypertensive medication or if a systolic blood pressure equal to 160 $\mathrm{mm} \mathrm{Hg}$ or over or a diastolic blood pressure of $95 \mathrm{~mm} \mathrm{Hg}$ or over was present in two measurements in the sitting position separated by a few minutes. Previous history of transient ischaemic attack or stroke was assessed by questionnaire and by analysis of clinical records. Angina pectoris as well as myocardial infarction were also assessed at baseline according to history and records on GPs files. The same procedure was adopted to investigate family history of stroke or myocardial infarction. Rheumatic fever was assessed by inquiry and review of medical records. Diabetes mellitus was diagnosed if subjects currently take oral antidiabetics or insulin. Current, former and never smoker was the classification of the smoking status. A similar classification was used for alcohol consumption and quantification was expressed in grams per day. Drinking pattern was recorded. Weight, height, waist and hip circumference were measured. An ECG was made and the diagnosis of arrhythmia, Q-wave myocardial infarction, and left ventricular hypertrophy was registered if present. A fasting venepuncture was performed to assess serum lipids, packed cell volume, $\gamma$-GT, platelets, white blood cells and lymphocytes. These laboratory procedures were dismissed if similar laboratory analysis from the past six months were available.

The methodology of case finding is based mainly on the information obtained from the GPs. Subjects and close family members are requested to inform personal family doctor if any event occur.

Transient ischaemic attack is defined as an acute loss of focal cerebral or vision function with symptoms lasting less than 24 hours and which after adequate investigation is presumed to be attributable to embolic or thrombotic vascular disease. Stroke is defined according to the WHO criteria as rapidly developing clinical signs of focal or global loss of cerebral function, with symptoms lasting more than 24 hours or leading to death, with no apparent cause other than that of vascular origin.

As soon as identified by GPs the incident fatal and non-fatal events information is immediately sent to our centre in the University Hospital of Coimbra to confirm the nature of the event. Confirmation is made by neurologists participating in the study by analysis of medical history or hospital discharge records or direct examination. Classification of stroke subtypes is made, when possible, with information obtained from imaging investigation. Regular mailings to GPs are sent every year to inquire about possible events not previously detected. Death certificates of all the patients dying during the study are centrally reviewed. Stroke events will be submitted for review to the EUROSTROKE Review board.

\section{Rotterdam, the Netherlands}

The Dutch contribution to EUROSTROKE comes from the Rotterdam Study, which is a population-based prospective follow up study among 7983 subjects, aged 55 years or over, living in the suburb of Ommoord in Rotterdam, the Netherlands. ${ }^{12}$ Baseline data were collected from March 1990 to July 1993. In the Rotterdam Study, information on incident fatal and non-fatal events is obtained from the GPs working in the study district of Ommoord as described earlier. ${ }^{13}$ The GPs involved report all possible cases of stroke to the Rotterdam research centre. Events are presented in coded information following the International Classification of Primary Care (ICPC).${ }^{14}$ With respect to the vital status of the participants, information is obtained at regular intervals from the municipal authorities in Rotterdam and also death of a participant is reported by GPs.

When an event or death has been reported, additional information is obtained by interviewing the GP and scrutinising information from hospital discharge records in case of admittance or referral. All suspected cerebrovascular events reported by the GPs were submitted for review to the EUROSTROKE case review board. From baseline to December 1994, 192 stroke cases were identified and submitted for review and a total of 384 control subjects were drawn from the remainder of the cohort that remained free from stroke during follow up. A total of 157 events were classified as definite or probable strokes.

Information on smoking, alcohol consumption, and medial history was obtained by questionnaire. The subject's smoking 
behaviour was categorised into current, former or never. Alcohol consumption was categorised into current drinkers and non-current drinkers (former and never). In addition, an estimate of grams of alcohol per day was obtained. Presence of diabetes mellitus was considered present when subjects used blood sugar lowering drugs. Information on a history of stroke was obtained by direct questioning at baseline: "Did you ever suffer from a stroke?". A similar approach was taken for myocardial infarction. Presence of angina pectoris was based on either the cardiovascular Rose questionnaire or direct questioning.

Systolic and diastolic blood pressure were measured twice at one occasion in sitting position. Height and weight were measured and body mass index $\left(\mathrm{kg} / \mathrm{m}^{2}\right)$ was calculated. An electrocardiogram was made and the presence of atrial fibrillation and of a Q-wave myocardial infarction was assessed by using the automated diagnostic classification system of the Modular Electrocardiogram Analysis System (MEANS)..$^{15}$ Left ventricular hypertrophy diagnosis was assessed on the parameters voltage, shape and repolarisation, as detailed elsewhere. ${ }^{15}{ }^{16}$ A non-fasting blood sample was taken for determination of serum lipids (total cholesterol, HDL cholesterol). Serum total cholesterol level was determined with an automatic analyser using enzymatic procedures in Rotterdam. $^{17}$

Fibrinogen levels were derived from the clotting curve of the prothrombin time assay using Thromborel S as reagent on an Automated Coagulation Laboratory. ${ }^{15}$ The latter method correlates well with the Von Clauss method..$^{18}$

Dietary intake was assessed with a 170 item semiquantitative food frequency questionnaire, which is a modified version of a previously validated questionnaire ${ }^{19}$ as described in detail elsewhere. ${ }^{20}$ The questionnaire was administered in two ways. In the first stage at the home of the participant, participants checked off foods they usually ate (at least twice a month) in the past year. The second stage at the research centre, a dietitian asked the participants how much and how often each food item was consumed. Furthermore, the questionnaire was thoroughly checked. Average daily dietary nutrient intake was calculated by multiplying the frequency and amount consumed for each food item by its nutrient content listed in an automated version of the Dutch Food Composition Table. ${ }^{21}$

\section{Novosibirsk, Russia}

The Russian contribution to EUROSTROKE comes from studies performed in the Octyabrsky, the Kirovsky and Leninsky districts of Novosibirsk, Siberia. The Novosibirsk cohort is based on three population-based surveys, that were conducted between 1984 and 1989 as part of the WHO MONICA project. ${ }^{23}$ The Novosibirsk cohort comprises 9006 men and women aged 25 to 64 years. Stroke cases were collected through a specifically developed stroke registry, aiming to identify fatal and non-fatal hospitalised and non-hospitalised stroke patients. ${ }^{23}{ }^{24}$ Stroke events were defined according to MONICA criteria and definitions. ${ }^{25}$ From baseline to December 1995, a total of 100 stroke cases had been identified and 200 control subjects were drawn from the database. Finally, 79 subjects proved to be true strokes.

Information on smoking, alcohol consumption, and medial history was obtained by questionnaire. The subject's smoking behaviour was categorised into current, former or never. Alcohol consumption was categorised into current drinkers and non-current drinkers (former and never). In addition, an estimate of grams of alcohol per day was obtained. Presence of diabetes mellitus was not determined. Information on a history of stroke was obtained by direct questioning at baseline: "Did you ever suffer from a stroke?". A similar approach was taken for myocardial infarction. Presence of angina pectoris was based on either the cardiovascular Rose questionnaire or direct questioning.
Systolic and diastolic blood pressure were measured twice at one occasion in sitting position. Height and weight were measured and body mass index $\left(\mathrm{kg} / \mathrm{m}^{2}\right)$ was calculated. An electrocardiogram was made and the presence of atrial fibrillation and of a Q-wave myocardial infarction and left ventricular hypertrophy were assessed according to the Minnesota classification system. A fasting blood sample was taken for determination of serum lipids (total cholesterol, HDL cholesterol). Serum total cholesterol level was determined with an automatic analyser using enzymatic procedures. ${ }^{26}$ Fibrinogen was not measured.

\section{EUROSTROKE CASE REVIEW BOARD}

The review board comprised of four Dutch neurologists. Based on all information, including symptoms and signs obtained by interviewing the GP or, in case of hospital referral, hospital data, the neurologists classified the events as definite, probable and possible stroke. Events were classified by two neurologists. In case of disagreement a third neurologist was consulted, whose opinion was decisive for the final classification. The present analysis is restricted to definite and probable events, outcomes in which a stroke most probably did occur according to the neurologist's opinion. For the present analysis an incident stroke was considered to have occurred when ( 1 ) the event had led to a hospitalisation and the hospital discharge record indicated a diagnosis of a new stroke. The clinical diagnosis was based on signs and symptoms, and neuroimaging investigations during hospital stay (definite stroke); or (2) in case of no hospitalisation, signs and symptoms associated with the event obtained from the general practitioner records and interview were highly suggestive of a stroke according to the neurologists (probable stroke) or (3) in case of out hospital death, when the general practitioners reported that the cause of death was a cerebrovascular accident and a cardiac cause was judged by the general practitioner to be highly unlikely (probable stroke).

The event was classified into first and recurrent stroke and into subarachnoid haemorrhage, intracranial haemorrhage, intracerebral infarction, and unspecified (not documented). For the non-MONICA centres (the Netherlands, United Kingdom) ischaemic stroke was further classified into lacunar infarct; total anterior circulation infarct; partial anterior circulation infarct; posterior circulation infarct and unspecified infarct (undocumented). The classification was made following the guidelines described by Bamford and coworkers ${ }^{27}$ and by the European Atrial Fibrillation Trial. ${ }^{28}$

\section{General data analytical approach}

The association between risk factor and stroke was evaluated using logistic regression techniques. For continuous variables like blood pressure or fibrinogen, generally analyses were performed using the risk factor as a continuous variable (per unit or standard deviation increase). Additional analyses were performed in quintiles or quartiles of the risk factor distribution as observed among controls. When the distribution of the risk factor differed considerably across centres, like in dietary intake, a Z score approach was being applied. Analyses were first performed within each centre. In the pooled analyses in which information of all centres was combined, each individual was categorised into the quartile based on the centre specific risk factor distribution. Adjustments were made for those variables that were related to both stroke risk and risk factor level, and not assumed to be an intermediate factor in the causal relation between risk factor and stroke occurrence. Whether the associations differed across centres, or between subgroups of patients, like diabetics or smokers, multiplicative interactions terms were used. 
Separate analyses were usually performed for total stroke, fatal and non-fatal stroke, cerebral infarction and haemorrhagic stroke, and when possible for subtypes of cerebral infarction. Results are usually expressed as odds ratios with corresponding 95\% confidence limits.

\section{DISCUSSION}

The description of the cohorts shows that across cohorts differences will exists in case ascertainment methods and in risk factor measurements. These difference may lead to bias in the association under study. Appropriate analytic approach will be to used to limit the possible biases. The approaches will be described in move detail in each paper where it is appropriate.

In summary, this paper describes the design and methodology of the participating cohorts in the EUROSTROKE project. Information is given about the cohort sampling, its size, the follow up procedures and event classification. Also information is given about the measurement of the cardiovascular and cerebrovascular risk factors in each of the cohorts separately.

\section{ACKNOWLEDGEMENTS}

Funding: EUROSTROKE is supported by grant BMHI-CT93-1786 from the European Community BIOMED I programme and by grant CIPD-CT94-0256 from the European Community PECO programme.

\section{Authors' affiliations}

M L Bots, D E Grobbee, Epidemiology and Biostatistics, Erasmus University Medical School, Rotterdam, the Netherlands

M L Bots, D E Grobbee, Julius Centre for General Practice and Patient Oriented Research, University Medical Centre Utrecht, Utrecht, the Netherlands

P C Elwood, Centre for Applied Public Health Medicine, University of Wales College of Medicine, Cardiff, UK

Y Nikitin, Russian Academy of Medical Sciences Siberian Branch, Institute of Internal Medicine, Novosibirsk, Russia

J T Salonen, Research Institute of Public Health, University of Kuopio, Kuopio, Finland

A Freire de Concalves, Neurology, Hospitais da Universidade de Coimbra, Coimbra, Portugal

D Inzitari, Neurological and Psychiatric Sciences, University of Florence, Florence, Italy

J Sivenius, Department of Neurology, Kuopio University Hospital, Kuopio, Finland

A Trichopoulou, Hygiene and Epidemiology, University of Athens Medical School, Athens, Greece

J Tuomilehto, Epidemiology and Health Promotion, National Public Health Institute, Helsinki, Finland

P J Koudstaal, Neurology, University Hospital Rotterdam Dijkzigt, Rotterdam, the Netherlands

\section{REFERENCES}

1 Grobbee DE, Koudstaal PJ, Bots ML, et al. Incidence and risk factors for ischaemic and haemorrhagic stroke in Europe. EUROSTROKE: A collaborative study among research centres in Europe: Rationale and design. Neuroepidemiology 1996;15:291-300.

2 The Caerphilly and Speedwell Collaborative Group. Caerphilly and Speedwell collaborative heart disease studies. J Epidemiol Community Health 1984;38:259-62

3 Rose GA, Blackburn H, Gillum RF, et al. Classification of the elctrocardiogram for population studies. In: Rose GA, Blackburn $\mathrm{H}$, eds. Cardiovascular survey methods. Geneva: World Health Organisation, 1968: 123-43.
4 Steele BW, Koehler DF, Kuba K, et al. An enzymatic approach to lipoprotein quantification. Am J Clin Pathol 1980;73:75-8.

5 Yarnell JWG, Fehily AM, Milbank JE, et al. A short dietary guestionnaire for use in an epidemiological survey; comparison with weighted dietary records. Hum Nutr Appl Nutr 1983:37A:103-12.

6 Salonen JT, Salonen R, Seppänen K, et al. High density lipoprotein, $\mathrm{HDL}_{2}$ and $\mathrm{HDL}_{3}$ subfractions and the risk of acute myocardial infarction: a prospective population study in Eastern Finnish men. Circulation 1992:84:129-39

7 Tuomilehto J, Sarti C, Narva EV, et al. The FinMonica stroke register. Community-based stroke registration and analysis of stroke incidence in Finland, 1983-1985. Am J Epidemiol 1992;135:1259-70.

8 Clauss A. Gerinnungsphysiologische schnellmethode zur bestimmung des fibrinogens. Acta Haematol 1957;17:237-46.

9 Ihanainen M, Salonen NR, Seppanen R, et al. Nutrition data collection in the Kuopio Ischaemic Heart Disease Risk Factor Study: nutrient intake of middle-aged Eastern Finnish men. Nutr Res 1989;9:597-604.

10 Riboli E. Nutrition and Cancer. Backgrounded rational of the European Prospective Investigation into Cancer and Nutrition (EPIC). Ann Oncol 1992;3:783-91.

11 Maggi S, Zucchetto M, Grigoletto F, et al, for the ILSA Group: The Italian Longitudinal Study on Aging (ILSA): design and methods. Aging Clin Exp Res 1994:6:464-73.

12 Hofman A, Grobbee DE, Jong PTVM de, et al. Determinants of disease and disability in the elderly. The Rotterdam Elderly Study. Eur J Epidemio 1991;7:403-22.

13 Bots ML, Hoes AW, Koudstaal PJ, et al. Common carotid intima-media thickness and risk of stroke and myocardial infarction. The Rotterdam Study. Circulation 1997;96:1432-7.

14 Lamberts H, Wood M, Hofmans-Okkes I. The international classification of primary care in the European Community. Oxford: Oxford University Press, 1991.

15 Van Bemmel JH, Kors JA, van Herpen G. Methodology for the Modular Electrocardiogram Analysis System (MEANS). Methods Inf Med 1990;29:346-53.

16 Willems JL, Abreu-Lima C, Arnaud P, et al. The diagnostic performance of computer programs for the interpretation of electrocardiograms. $N$ Engl J Med 1991;325:1767-73.

17 VanGent CM, VanderVoort HA, de Bruijn AM, et al. Cholesterol determinations. A comparative study of methods with special reference to enzymatic procedures. Clin Chem Acta 1977:75:243-51.

18 Rossi E, Mondonico P, Lombardi A, et al. Methods for determination of functional (clottable) fibrinogen by the new ACL coagulometers. Thromb Res 1988;52:453-68.

19 Van der Brandt PA, Goldbohm RA, van 't Veer P, et al. A large scale prospective cohort study on diet and cancer in the Netherlands. J Clin Epidemiol 1990;43:285-95

20 Jama JW, Launer L, Witteman JCM, et al. Dietary antioxidants and cognitive function in a population-based sample of older persons. The Rotterdam Study. Am J Epidemiol 1996;144:275-80.

21 Stichting Nederlands Voedingsstoffenbestand. NEVO tabel 1993. Den Haag, the Netherlands: 1993

22 WHO MONICA Project Investigators. World Health Organization MONICA Project (monitoring trends and determinants in cardiovascular disease): a major international collaboration. J Clin Epidemiol 1988:41:105-14.

23 Asplund K, Tuomilehto J, Steygmar B, et al. Diagnostic criteria and quality control of the registration of stroke events in the MONICA Project. Acta Med Scand 1988;728 (suppl):26-39.

24 Feigin VL, Wiebers DO, Whisnant JP, et al. Stroke incidence and 30-day case fatality rates in Novosibirsk, Russia. Stroke 1995;26:924-9

25 Thorvaldsen P, Asplund K, Kuulasmaa K, et al. Stroke incidence, case fatality, and mortality in the WHO MONICA Project. Stroke 1995;26:361-7.

26 US National Institute of Health. Manual of laboratory operations of the lipid research clinics program. Lipids and lipoprotein analysis. Washington DC: Government Printing Office Publication. No NIH 1974 75-628.

27 Bamford J, Sandercock P, Dennis M, et al. Classification and natural history of clinically identifiable subtypes of cerebral infarction. Lancet $1991 ; i: 1521-6$.

28 European Atrial Fibrillation Trial Study Group. Secondary prevention in non-rheumatic atrial fibrillation after transient ischaemic attack or mino stroke. Lancet 1993;342:1255-62. 